\title{
Increased Risk of Mortality in Alzheimer's Disease Patients with More Advanced Educational and Occupational Attainment
}

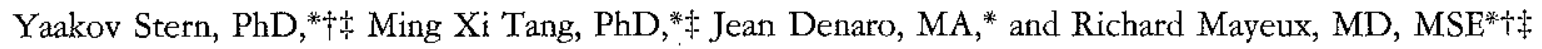

\begin{abstract}
A reserve hypothesis suggests that clinical symptoms of Alzheimer's disease (AD) begin earlier in individuals with less education. Therefore, patients with less education might survive longer after diagnosis than those with more education. Two hundred forty-six subjects with probable AD were followed for 1 to 4 years. There were 78 deaths; 30 deaths occurred in the 127 patients whose education was $\leq 8$ years, while 48 deaths occurred in the 119 patients with $>8$ years of education. Cox proportional hazards models adjusted for age, gender, and clinical dementia rating (CDR) showed that patients with more education had increased mortality (continuous variable: $R R=1.06$ for each year of education; $95 \%$ confidence interval $[\mathrm{CI}], 1.01-1.11$; dichotomous variable at $8 \mathrm{yr}: R R=1.76$; $\mathrm{CI}, 1.11-2.77$ ). This observation might at first seem counterintuitive, since groups with lower socioeconomic status are often at greater mortality risk. It implies that at any level of assessed clinical severity, the underlying pathology of AD is more advanced in patients with more education, resulting in shorter duration of diagnosed disease before death. These findings suggest either that education systematically influences global ratings of disease severity or that education provides a reserve against the clinical manifestation of $\mathrm{AD}$ pathology.
\end{abstract}

Stern Y, Tang MX, Denaro J, Mayeux R. Increased risk of mortality in Alzheimer's disease patients with more advanced educational and occupational attainment. Ann Neurol 1995;37:590-595

It has been hypothesized that educational and occupational attainment (EOA) may influence the clinical expression of Alzheimer's disease (AD) [1-4]. Several investigators have reported higher prevalence of dementia in elders with lower EOA [5-10]. Although one group reported no relation between EOA and incident dementia [11], we have reported that lower EOA is associated with increased incidence [12]. We have hypothesized that EOA provides a reserve against dementia, in that patients with higher EOA can cope with advancing disease pathology more effectively by maintaining function longer. If this is the case, for any level of clinical severity, AD pathology would be more advanced in patients with higher EOA. This hypothesis led us to investigate rates of mortality in patients with $\mathrm{pAD}$ as a function of EOA. We predicted that in patients matched for clinical severity, higher EOA would be associated with more rapid mortality.

\section{Materials and Methods}

\section{Subjects}

Data were obtained from a study of dementia in the elderly who reside in the Washington Heights and Inwood communities of New York City. We developed a registry of patients with $A D$ based on referrals and screening of elders from regional hospitals (including inpatient and outpatient services), private practitioners in the community, federal and state health agencies, health maintenance organizations, and senior centers. Thus, the registry attempted to identify all prevalent cases of dementia in the community. Nondemented elders from the community were also recruited and followed as controls. The Columbia University institutional review board reviewed and approved this project. All subjects provided written informed consent.

The study design required that subjects be evaluated yearly. Subjects included in the current analyses met the following criteria: (1) They met criteria for probable AD at the baseline diagnostic evaluation; (2) they had not had an acute stroke or Parkinson's disease; and (3) they had at least one follow-up evaluation and/or reliable information was available for the date of subsequent death.

To investigate the potential effect of educational and occupational attainment on survival in nondemented individuals, subjects who were not demented at the baseline diagnostic evaluation were included as a comparison group. This group met the other inclusion criteria described above.

\section{Diagnostic Evaluation}

All subjects received the same standardized diagnostic evaluation. Evaluations were conducted in either English or Spanish, based on the subjects' primary language and their opinion of which language would yield better performance. The diag-
From the Departments of *Neurology and PPsychiatry, Columbia University, College of Physicians and Surgeons, and the $\$$ Gertrude H. Sergievsky Center, New York, NY.
Received Jun 7, 1994, and in revised form Sep 19 and Dec 5. Accepted for publication Dec 8, 1994.

Address correspondence to Dr Stern, Sergievsky Center, 630 West 168th Street, New York NY 10032. 
nostic evaluation has been described previously $[12,13]$. It included a neuropsychological test battery, a standardized medical/neurological evaluation, and assessments of functional capacity and mood.

Information from all of these evaluations was presented at a diagnostic conference of physicians and neuropsychologists, and a consensus diagnosis was made. The diagnosis of dementia was based on Diagnostic and Statistical Manual of Mental Disorders (3rd edition, revised) criteria [14] and required evidence of cognitive deficit, based on the neuropsychological scores, as well as evidence of impaiment in social or occupational function, based on the formal functional assessments, elicited history, or both. In addition, aspects of physicians' examination or history and psychiatric evaluations were evaluated to determine the type of dementia present. For the diagnosis of probable or possible Alzheimer's disease, we used the criteria of the National Institute of Neurological and Communicative Disorders and Stroke-Alzheimer's Disease and Related Disorders Association [15]. A Clinical Dementia Rating (CDR) [16] was assigned to reflect severity.

\section{Occupation}

At the initial visit, the subject's primary occupation was recorded and classified based on the following US census categories: student, housewife, unskilled/semiskilled, skilled trade or craft, clerical/office worker, manager business/government, and professional/technical. A housewife who had been employed for a significant period of her adult life (i.e., more than $10 \mathrm{yr}$ ) was classified according to that occupation.

\section{Mortality}

We often received mortality information from the original referral source (e.g., nursing home, physician). We learned of other subjects' deaths when attempting to complete annual follow-up. For patients who could not be contacted for follow-up and were otherwise lost to follow-up, death information was obtained as available through the National Death Index.

\section{Statistical Analysis}

We used Cox proportional hazards models [17] to assess relative risk $(R R)$ of mortality associated with education and occupation. The Cox analyses were structured to evaluate the time from the initial evaluation to death. Patients who did not die were treated as censored observations from the time of their last follow-up evaluation. For the initial analysis of the $R R$ of death associated with education, years of education was treated as a continuous variable. To simplify interpretation of $R R$ s associated with education and of possible interactions between education and other variables, education was then dichotomized into low ( 8 or less years) and high (more than 8 years) for all subsequent analyses. For the analysis of occupation, subjects who were classified as housewives only $(n=44)$, other $(n=9)$, or unknown ( $\mathrm{n}$ $=3$ ) were omitted, since these classifications could not be fit directly into accepted social class hierarchies. The remaining classifications were grouped into low (unskilled/semiskilled, skilled trade or craft, and clerical/office worker) and high (manager business/government and professional/technical) occupational levels.
The baseline CDR score was included as a covariate in the Cox analyses to control for disease severity. The CDR was selected since this measure stages patients based on a group of functional domains rather than on performance on a particular instrument. Based on preliminary analyses, the CDR rating was dichotomized into mild $(C D R=1)$ and moderate to terminal $(C D R>1)$. Age at the initial visit and gender were also included as covariates in all analyses.

Initially, the $R R$ s associated with low education and low occupation were analyzed separately. To evaluate potential interactions of risk associated with education and occupation, these variables and their interaction were then included in the same Cox model. In addition, subjects were divided into the following four groups: low education and occupation, high education and low occupation, low education and high occupation, high education and high occupation and separate Cox analyses conducted using subjects with high education and occupation as the reference group.

\section{Results}

\section{Subject Accrual and Follow-up}

There were 448 subjects who met criteria for pAD at the baseline evaluation. Basic demographic data are summarized in Table 1. Follow-up data were available for 246 patients. Of the remaining 202 patients, 127 were not yet due for their 1-year follow-up, 20 refused follow-up, 23 could not be located, 5 moved out of the study area, and 25 had an evaluation pending that had not yet been scheduled. The subjects who were and were not followed did not differ in educational or occupational attainment. However, the subjects who were not followed were slightly but significantly younger, and their dementia severity, based on CDR, short Blessed, and Blessed Dementia Rating Scale (BDRS) was slightly but significantly lower (see Table 1).

In the 246 patients for whom follow-up data were available, there were 78 deaths. The distribution of demographic variables in patients who did and did not die are presented in Table 2 . Immediate cause of death

Table 1. Comparison of Patients Who Were and Were Not Followed

\begin{tabular}{lcll}
\hline & $\begin{array}{l}\text { Followed } \\
\text { (SD) }\end{array}$ & $\begin{array}{l}\text { Not Followed } \\
\text { (SD) }\end{array}$ & $p<$ \\
\hline $\mathrm{n}$ & 246 & 202 & \\
Age at intake (yr) & $83.9(7.5)$ & $81.3(8.2)$ & 0.01 \\
Gender (M/F) & $61 / 185$ & $49 / 153$ & $\mathrm{NS}$ \\
Education (yr) & $6.9(4.4)$ & $6.9(4.5)$ & $\mathrm{NS}$ \\
Education (low/high) & $168 / 78$ & $138 / 64$ & $\mathrm{NS}$ \\
Occupation (low/high) & $163 / 22$ & $141 / 18$ & $\mathrm{NS}$ \\
CDR (1/>1) & $122 / 124$ & $149 / 53$ & 0.01 \\
Short Blessed & $17.9(7.2)$ & $16.1(7.8)$ & 0.05 \\
BDRS & $7.4(5.1)$ & $5.1(4.4)$ & 0.01 \\
\hline
\end{tabular}

$p$ values are for $t$ tests or $\chi^{2}$ tests as appropriate.

$\mathrm{SD}=$ standard deviation; CDR = Clinical Dementia Rating; BDRS $=$ Blessed Dementia Rating Scale. 
Table 2. Comparison of Patients Who Did and Did Not Die

\begin{tabular}{lcll}
\hline & $\begin{array}{l}\text { Did Not Die } \\
\text { (SD) }\end{array}$ & $\begin{array}{l}\text { Died } \\
\text { (SD) }\end{array}$ & $p<$ \\
\hline $\mathrm{n}$ & 168 & 78 & \\
Age at intake (yr) & $82.9(7.3)$ & $86.1(7.3)$ & 0.01 \\
Gender (M/F) & $35 / 133$ & $26 / 52$ & 0.05 \\
Education (yr) & $6.3(4.1)$ & $8.3(4.9)$ & 0.01 \\
Education (low/high) & $125 / 43$ & $43 / 35$ & 0.01 \\
Occupation (low/high) & $114 / 10$ & $49 / 12$ & 0.05 \\
CDR (1/>1) & $104 / 64$ & $18 / 60$ & 0.01 \\
Short Blessed & $16.8(6.9)$ & $20.9(7.1)$ & 0.01 \\
BDRS & $6.0(4.7)$ & $10.3(4.9)$ & 0.01 \\
\hline
\end{tabular}

$p$ values are for $t$ tests or $\chi^{2}$ tests as appropriate.

$\mathrm{SD}=$ standard deviation; $\mathrm{CDR}=$ Clinical Dementia Rating; BDRS $=$ Blessed Dementia Rating Scale.

was available for 39 subjects and included pneumonia ( 4 in the low education group/ $/ 2$ in the high education group), other infection (1/0), myocardial infarction $(5 / 3)$, other cardiovascular (3/3), other cardiopulmonary (4/4), cerebrovascular (0/1), Alzheimer's disease $(0 / 1)$, and other $(5 / 3)$. Thus, the distribution of causes of death appeared comparable in the high and low education groups.

Cox Analyses

The predictive effects of gender, CDR, education, and occupation were evaluated with separate Cox proportional hazards models. A higher $R R$ of death was associated with male gender $(R R=2.0 ; 95 \%$ confidence interval $[\mathrm{CI}], 1.2-3.2), \mathrm{CDR}>1(R R=3.2 ; 95 \%$ $\mathrm{Cl}, 1.9-5.5)$, more years of education $(R R=1.1$;

Fig 1. Survival curve comparing cumulative survival in Alzbeimer's disease patients with bigh $(>8 y r)$ and low $(\leq 8 y r)$ of education. Curves are based on Cox analyses, which control for age, gender, and Clinical Dementia Rating.
1.0-1.1), and higher occupational attainment $(R R=$ $2.2 ; 1.1-4.3$ ). When education was dichotomized into 8 or less years and 9 years or more, greater education was also associated with an increased $R R$ of death $(R R$ $=1.9 ; 1.2-3.0$ ).

When CDR, education, gender, and age at the initial visit were simultaneously included in a Cox model, all but age made significant contributions. As expected, individuals with $\mathrm{CDR}>1$ had an increased tisk of mortality $(R R=3.1 ; 95 \% \mathrm{CI}, 1.8-5.3)$. Men had increased risk of mortality $(R R=1.8 ; 1.08-2.9)$. Age was not associated with significantly increased risk $(R R$ $=1.0 ; 0.9-1.1)$. Finally, patients with more years of education had increased risk $(R R=1.1 ; 1.01-1.2)$. When education was treated as a dichotomous variable in the model, greater education was also associated with an increased $R R$ of death $(R R=1.8 ; 1.1-2.8)$ (Fig 1).

In a similar way, when CDR, occupation gender, and age were simultaneously included in a Cox model, all made significant contributions. Individuals with $C D R$ $>1$ had an increased risk of mortality $(R R=2.9 ; 95 \%$ $\mathrm{Cl}, 1.6-5.2)$. Men had increased risk of mortality $(R R$ $=2.1 ; 1.3-3.6)$ as did older subjects $(R R=1.04$; 1.0-1.1). Finally, patients with higher occupational attainment had increased risk $(R R=2.0 ; 95 \% \mathrm{CI}$, 1.0-3.9) (Fig 2).

To evaluate the differential contribution of education and occupation to the prediction of mortality, education, occupation, and their interaction were included in a forward stepwise Cox regression model that controlled for age, CDR, and gender. In this analyses, education remained significant $(R R=2.3 ; 95 \% \mathrm{CI}$, 1.4-3.9), but occupation and the interaction of education and occupation did not enter into the model. We also divided patients into four education/occupation

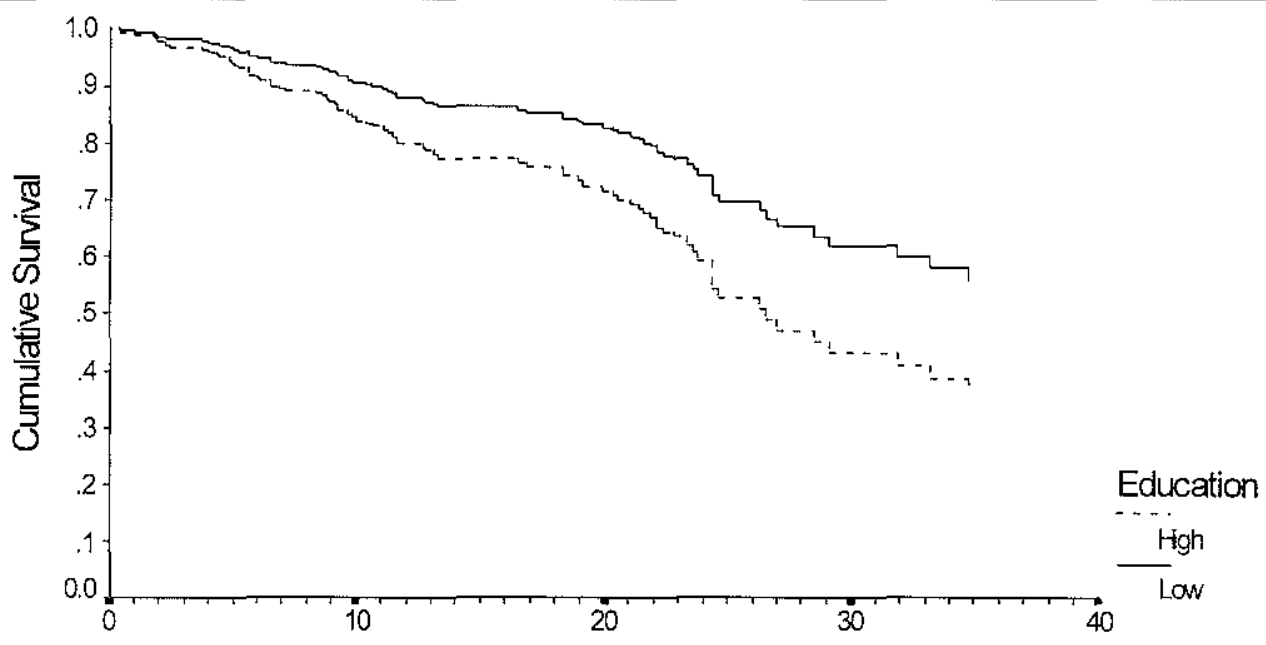

Months from first evaluation 


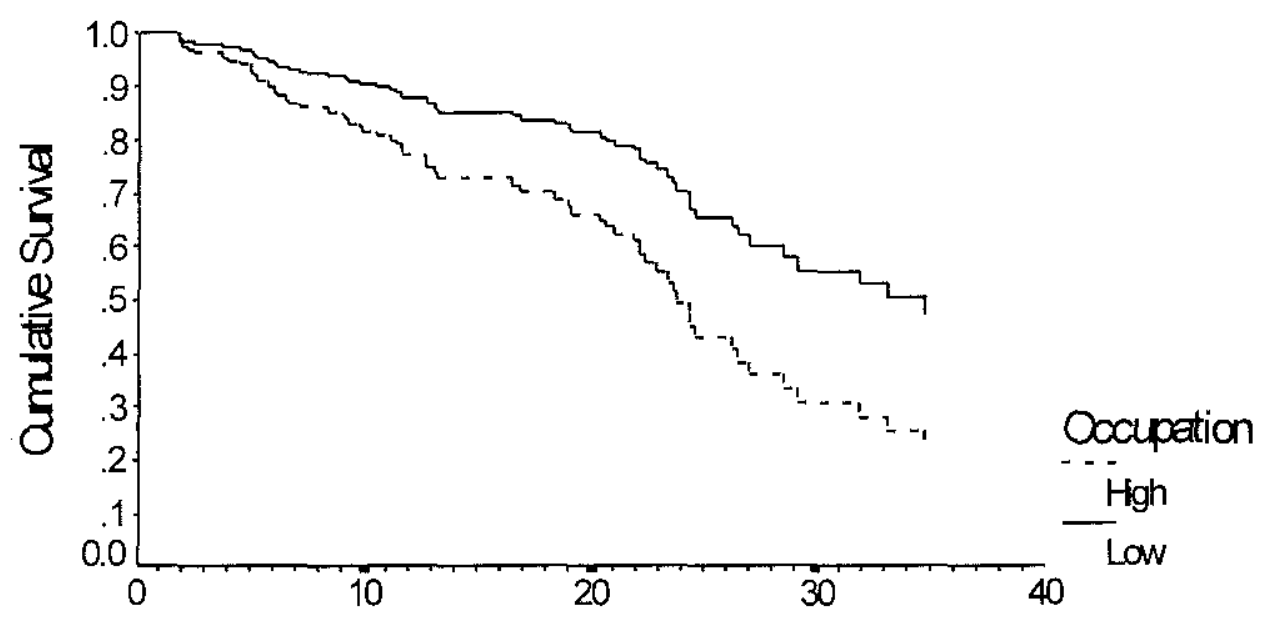

Months from first evaluation

Fig 2. Survival curve comparing cumulative survival in Alzbeimer's disease patients with bigh and low occupational attainment. Cumes are based on Cox analyses, which control for age, gender, and Clinical Dementia Rating.

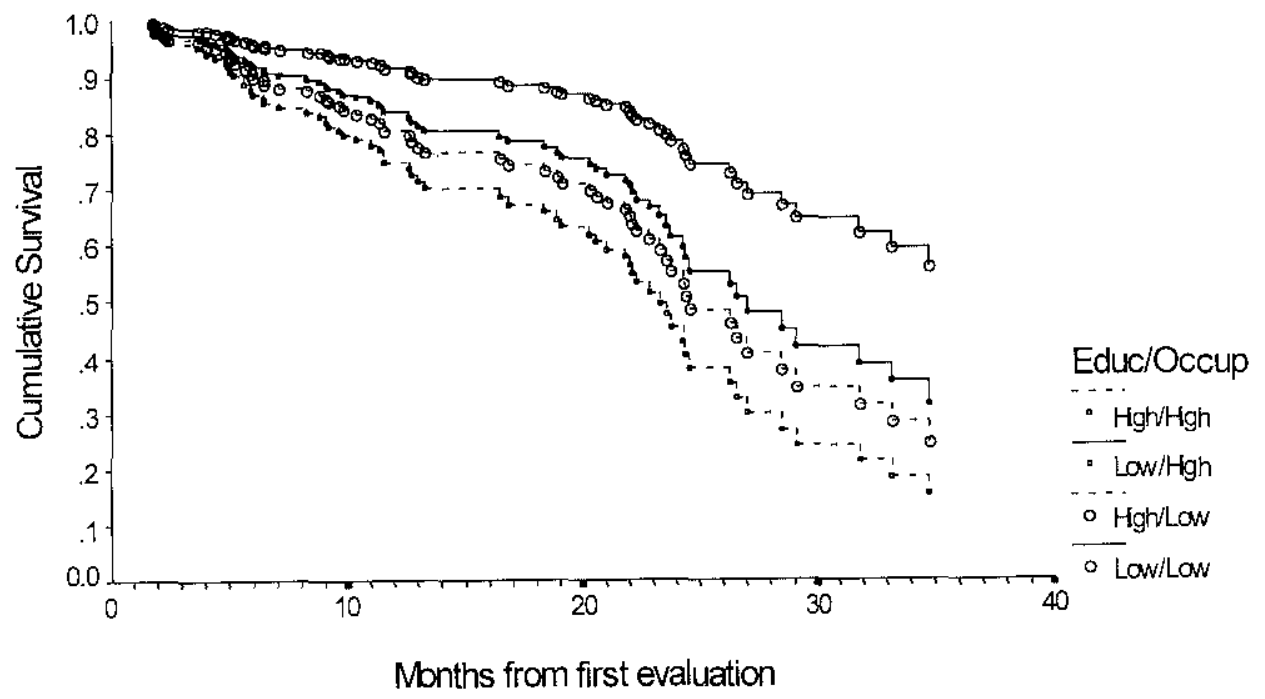

Fig 3. Survival curve comparing cumulative survival in Alzbeimer's disease patients classified into four groups based on educational and occupational attainment. Curves are based on Cox analyses, which control for age, gender, and Clinical Dementia Rating.

groups. There were 113 subjects in the low education/ occupation group, 50 with high education and low occupation, 5 with low education and high occupation, and 17 with both high education and occupation. Results are illustrated in Figure 3. In comparison with the patients with low education and occupation, those with high education and occupation had significantly elevated risk of mortality ( $R R=3.2 ; 95 \% \mathrm{CI}, 1.5-7.0)$. The high education/low occupation group also had sig-

nificantly elevated risk of mortality $(R R=2.4 ; 95 \%$ CI, 1.4-4.3), while the $R R$ in the low education/high occupation group was not significant $(R R=2.0 ; 95 \%$ $\mathrm{CI}, 0.5-8.7)$. The latter result may, in part, be due to the small number of subjects in that group.

We evaluated the possibility that in the patients with a CDR rating greater than 1 , dementia was actually more severe in those with higher education or occupation attainment. Using $t$ tests, there was no significant difference between the low and high education groups with CDR ratings greater than 1 on three selected measures included in the diagnostic battery, i.e., mental status as assessed with the short version of the Blessed 
Memory Information and Concentration test $\{18,19\}$, functional capacity as rated by the BDRS (Part 1) [19], and memory as measured by total recall on the selective reminding test [20]. In a similar way, there was no significant difference between the low and high occupation groups with CDR ratings greater than 1 on these measures.

We also included the Blessed Memory Information and Concentration test score as an additional covariate in the Cox analyses described above, which also controlled for age, gender, and CDR. Results were comparable. Higher education remained associated with a higher risk of mortality when education was treated either as continuous $(R R=1.09 ; 95 \% \mathrm{CI}, 1.03-1.15)$ or a dichotomous variable $(R R=2.06 ; 95 \% \mathrm{CI}$, 1.23-3.46). Using a similar model, the relative risk of mortality associated with occupational attainment was of borderline significance $(R R=2.06 ; 95 \% \mathrm{CI}$, $0.95-4.45)$. In the four education/occupation groups, compared with patients with low educational and occupational attainment, those with high education and occupation had an elevated risk of mortality $(R R=3.97$; $95 \% \mathrm{CI}, 1.57-10.08)$. The high education/low occupation group also had an elevated risk $(R R=2.76 ; 95 \%$ $\mathrm{CI}, 1.44-5.32$ ), while the RR in the low education/ high occupation group was not significant $(R R=1.97$; $95 \% \mathrm{CI}, 0.43-8.92)$.

\section{Nondemented Comparison Group}

There were 292 individuals in the nondemented comparison group. Average age was 72.6 years $(\mathrm{SD}=8.7$ yr). There were 93 men and 199 women. The average years of education was 10.8 ( $\pm 4.2 \mathrm{yr}$ ); 94 had 8 or less years of education. Occupational attainment was classified as high in 214 subjects and low in 246; the remaining subjects were housewives.

There were 37 deaths. In Cox analyses that controlled for age and gender, there was no significant effect of higher educational attainment on survival whether education was treated as a continuous $(R R=$ $0.9 ; 95 \% \mathrm{CI}, 0.9-1.1)$ or a dichotomous variable $(R R$ $=1.4 ; 95 \% \mathrm{CI}, 0.6-2.9)$. In a similar way, there was no effect of higher occupational attainment on survival $(R R=0.6 ; 95 \% \mathrm{CI}, 0.3-1.3\rangle$.

\section{Discussion}

The observation that higher EOA is associated with more rapid mortality might at first seem counterintuitive, since lower socioeconomic status groups often are at greater mortality risk due to issues such as medical care and living conditions. However, this observation lends support to the concept that $\mathrm{AD}$ is expressed earlier (i.e., when $\mathrm{AD}$-related neuropathologic changes are less advanced) in patients with lower EOA. Thus, survival time is increased.

The reserve hypothesis would predict that educa- tional attainment should correlate inversely with severity of $\mathrm{AD}$ histopathology given comparable clinical severity of dementia. We have tested this prediction in a separate set of studies of patients with established diagnosis of $\mathrm{AD}$. Using xenon regional cerebral blood flow, we assessed perfusion in the parietotemporal area as an index of $\mathrm{AD}$ pathology. In patients with comparable clinical severity of dementia, both increased years of education and higher occupational attainment were associated with more parietotemporal perfusion deficit, supporting our hypotheses [4, 21].

The mechanism by which occupational experience might contribute to reserve against $\mathrm{AD}$ is unclear. This reserve could be the result of increased synaptic density in neocortical association cortex acquired on the basis of stimulation [1], or an acquired set of skills or repertoires $[2,3,21]$. The latter possibility is more compatible with our present data, since it could explain how reserve protects against the expression of $\mathrm{AD}$ pathology but not against death. It also explains our previous observation that features of occupational experience such as physical demands are associated with reserve [21]. Presumably, aspects of life experience could modify the paradigms used by the brain to mediate a task in a way that would make the paradigms more efficient or resilient in the face of $\mathrm{AD}$ pathology.

The concept of reserve must be weighed against the alternate possibility of an assessment bias. Assessment bias may result in patients with more education performing slightly better on standardized evaluations than those with less education despite comparable disease pathology. Our decision to control for dementia severity using the CDR rating was an attempt to minimize assessment bias. This rating is only partially dependent on cognitive performance and is driven primarily by the patient's functional performance in several contexts. Thus, it might be logical to assume that it would be less likely for patients to perform better on this scale simply because they had higher educational or occupational attainment. With regard to more basic activities such as eating and toileting, there would probably be little debate on this issue. Instrumental activities of daily living involve more of a cognitive component, so educational or occupational attainment may influence performance on these measures as well. There is also evidence that increased educational attainment may influence functional decline in normal aging [22].

It is difficult to differentiate between cognitive reserve and assessment bias in the present study. However, to the extent that the CDR assesses behaviors relevant to $\mathrm{AD}$ this discrimination becomes somewhat artificial because (1) either explanation predicts preserved function, and (2) for any level of functional impairment patients with higher EOA will have more advanced $A D$ pathology. In effect, the assessment bias in 
this study would simply be that higher EOA subjects were rated as less demented on a measure that assesses a wide range of AD-related changes. Since this "bias" reflects that subject's ability to perform better across a wide range of behaviors in the face of $\mathrm{AD}$, it is synonymous with reserve.

Our analyses implicitly assume that there is a relationship between death and the severity of $\mathrm{AD}$ pathology and that our subjects died of $\mathrm{AD}$. These assumptions are generally warranted except in cases where some other acute medical event intervenes. Two of our current observations argue against the idea that differential mortality in the high EOA AD patients was a function of some other disease process. First, the distribution of causes of death was similar in high and low education $A D$ groups and provides no alternate explanation for the different mortality rates. Second, a relation between mortality and EOA was not noted in the controls. This minimized the possibility that some other EOA-related factor accounts for differential mortality in the AD group.

To our knowledge, the relation of EOA and mortality has not been studied in $\mathrm{AD}$. However, our finding of increased mortality risk associated with more severe disease has been inconsistently observed in previous studies. A study of the 1,259 inhabitants of Leiden, aged 85 and over, found a mortality rate ratio $(M R R)$ of 1.9 for the demented compared with nondemented groups [23]. No difference in mortality rate was found between patients with mild vs moderate to severe dementia. The East Boston group [24] reported that, overall, risk of death associated with $\mathrm{AD}$ was only moderately increased over that of unaffected individuals $(M R R=1.44)$. Risk of death was greatest for patients with severe cognitive impairment or cachexia, both markers of advance disease; while less severely affected patients did not have elevated risk.

In summary, our findings suggest that for any level of $\mathrm{AD}$ pathology, the clinical expression of $\mathrm{AD}$ is less severe in patients with more advanced EOA. Therefore, for any level of clinical expression, patients with more advanced EOA are more likely to die sooner.

Supported by Federal grants AG07232, AG07370, and RR00645 and The Charles S. Robertson Memorial Gift for Alzheimer's Disease from the Banbury Fund.

\section{References}

1. Katzman R. Education and the prevalence of dementia and Alzheimer's disease. Neurology 1993;43:13-20

2. Mortimer JA. Do psychosocial risk factors contribute to Alzheimer's disease? In: Henderson AS, Henderson JH, eds. Etiology of dementia of Alzheimer's type. New York: John Wiley and Sons, 1988:39-52

3. Gurland BJ. The borderiands of dementia: the influence of sociocultural characteristics on rates of dementia occurring in the senium. Aging 1981;15:61-84

4. Stern Y, Alexander GE, Prohovnik I, Mayeux R. Inverse rela- tionship between education and parietotemporal perfusion deficit in Alzheimer's disease. Ann Neurol 1992;32:371375

5. Zhang MY, Katzman R, Jin H, et al. The prevalence of dementia and Alzheimer's disease (AD) in Shanghai China: impact of age, gender and education. Ann Neurol 1990;27:428-437

6. Dartigues JF, Gagnon M, Miche P, et al. Le programme de recherche paquid sur l'epidémiologie de la démence méthodes et résultats initiaux. Rev Neurol (Paris) 1991;147:225-230

7. Bonaiuto S, Rocca WA, Lippi A, et al. Impact of education and occupation on prevalence of Alzheimer's disease (AD) and multi-infarct dementia (MID) in Appignano, Macerata Province, Italy. Neurology 1990;40(suppl 1):346 (Abstract)

8. Fratiglioni L, Grut M, Forsell Y, et al. Prevalence of Alzheimer's disease and other dementias in an elderly urban population: relationship with age, sex and education. Neurology 1991;41: 1886-1892

9. Sulkava R, Wikström J, Aromaa, et al. Prevalence of severe dementia in Finland. Neurology 1985;35:1025-1029

10. Korczyn AD, Kahana E, Galper Y. Epidemiology of dementia in Ashkeion, Israel. Neuroepidemiology 1991;10:100 (Abstract)

11. Beard CM, Kokmen E, Offord K, Kurland LT. Lack of association between Alzheimer's disease and education, occupation, marital status, or living arrangement. Neurology 1992;42:20632068

12. Stern $Y$, Gurland B, Tatemichi T, et al. Influence of education and occupation on the incidence of Alzheimer's disease. JAMA 1994;271:1004-1010

13. Stern Y, Andrews H, Pittman J, et al. Diagnosis of dementia in a heterogeneous population: development of a neuropsychological paradigm-based diagnosis of dementia and quantified correction for the effects of education. Arch Neurol 1992;49:453-460

14. American Psychiatric Association. Diagnostic and statistical mantual of mental disorders. 3rd ed, revised. Washington, DC: APA, 1987

15. McKhann G, Drachman D, Folstein M, et al. Clinical diagnosis of Alzheimer's disease: report of the NINCDS-ADRDA work group under the auspices of the Department of Health and Human Services task force on Alzheimer's disease. Neurology 1984;34:939-944

16. Hughes CP, Berg L, Danziger WL, et al, A new clinical scale for the staging of dementia. Br J Psychiatry 1982;146:566-572

17. Lawless JF. Statistical model and methods for lifetime data. New York: John Wiley and Sons, Inc, 1982

18. Katzman R, Brown T, Fuld P. Validation of a short orientationmemory-concentration test of cognitive impairment. Am J Psychiatry $1983 ; 140: 734-738$

19. Blessed G, Tomlinson BE, Roth M. The association between quantitative measures of dementia and of senile changes in the cerebral grey matter of elderly subjects. Br J Psychol 1968;225: $797-811$

20. Buschke H, Fuld PA. Evaluating storage, retention, and retrieval in disordered memory and learning. Neurology 1974;24:10191025

21. Stern Y, Alexander GE, Prohovnik I, et al. Relationship between lifetime occupation and parietotemporal flow: implications for a reserve against Alzheimer's disease pathology. Neurology 1995;45:55-60

22. Snowdon DA, Ostwald SK, Kane RL. Education, survival, and independence in elderly Catholic sisters, 1936-1988. Am J Epidemiol 1989;130:999-1012

23. Heeren TJ, van Hemert AM, Rooymans HGM. A communitybased study of survival in dementia. Acta Psychiatr Scand 1992; $85: 415-418$

24. Evans DA, Smith LA, Scherr PA, et al. Risk of death from Alzheimer's disease in a community population of older persons. Am J Epidemiol 1991;134:403-412 